\title{
Frontal lesions and the rate of operant behavior in the rat
}

\author{
R. C. WILCOTT \\ Case Western Reserve University, Cleveland, Ohio
}

\begin{abstract}
Results previously obtained by the method of electrical stimulation indicate that the frontal pole and adjacent cortex of the rat can have an inhibitory influence on the rate of barpressing for food. The effects of bilateral lesions in this cortical area of the rat on the rate of barpressing were investigated. When lesioned rats were hungry, neither their rate of barpressing for food nor their rate of responding during extinction was increased as compared with control rats. When lesioned rats were allowed ad-lib feeding in the home cage, the rate of barpressing for food was also not increased, but the rate of responding during extinction was increased. Stabilimeter activity of these rats was increased when they were hungry, but not when they were allowed ad-lib feeding in the home cage. It is concluded that these frontally lesioned rats displayed a perseveration tendency in their operant behavior, but that this tendency was counteracted by the effects of increased general activity when they were hungry.
\end{abstract}

Electrical stimulation, particularly at a low frequency, in parts of the frontal cortex of the rat and cat suppresses autonomic and skeletal activity (e.g., Glaser \& Griffin, 1962; Siegle \& Wang, 1974). In these studies, stimulation appears to have activated cells in the frontal cortex that have an inhibitory influence on behavior. If this interpretation is correct, bilateral lesions in appropriate parts of the frontal cortex should destroy many of these inhibitory cells, which in turn should produce an increase in autonomic and skeletal activity. Such an effect has usually been found.

With regard to autonomic activity, stimulation in the prefrontal cortex of the cat suppresses skin potential responses to auditory stimuli (Wang \& Brown, 1956; Wilcott, 1974) and prefrontal lesioning enhances skin potential responses (Murray \& Wang, 1967; Wilcott, 1967). Furthermore, stimulation in parts of the frontal cortex of the rat reduces heart rate response to a noxious stimulus, and lesions in the same frontal area increase heart rate activity (Glaser \& Griffin, 1962).

With regard to skeletal activity, running in an activity wheel can be suppressed by stimulation in or near the frontal pole of the rat (Wilcott, 1979), and running is increased following lesioning in the frontal poles (Campbell \& Lynch, 1969). In addition, stimulation in the frontal pole has no effect on the general activity of rats as measured in a stabilimeter (Wilcott, 1979), and frontal pole lesions also have little or no effect on stabilimeter activity, except during food deprivation (Campbell \& Lynch, 1969).

The author's mailing address is: Department of Psychology, Case Western Reserve University, Cleveland, Ohio 44106.
An exception to the apparent agreement between the behavioral effects of stimulation and lesions in the frontal cortex is the effects of these procedures on the rate of operant behavior. Stimulation in the frontal pole region of the rat can completely suppress barpressing for food (Wilcott, Sabol, \& Yurcheshen, 1976). But results obtained following frontal lesions are inconsistent. Lesions in the frontal pole of the rat decrease the rate of barpressing with a variableinterval schedule (Campbell \& Lynch, 1969). But, in the rat, large dorsofrontal lesions that apparently include the frontal pole have little effect on barpressing with a continuous-reinforcement (CRF) schedule (Schwartz \& Isaacson, 1972). In addition, large dorsofrontal lesions in the rat do not affect the rate of responding during extinction (Schwartz \& Isaacson, 1972). Furthermore, medial or orbital frontal lesions do not affect the rate of barpressing of the rat with a CRF schedule (Kolb, Nonneman, \& Singh, 1974). But in that study, orbital lesions did produce a significant increase in the rate of responding during extinction.

It appears that the location of lesions and the testing procedure may be important factors in the effects of frontal lesions on the rate of operant behavior. The purpose of this study was to investigate this problem further. It is convenient to describe these observations as two separate experiments.

\section{EXPERIMENT 1}

The aim of Experiment 1 was to test the effects of bilateral frontal lesions on the rate of barpressing for food and the rate of responding during extinction. Lesions were made in parts of the frontal cortex where low-frequency stimulation had been found to 
suppress barpressing for food. The dorsolateral, orbital, and medial cortex of the rat have been mapped for sites where stimulation suppresses barpressing (Wilcott, 1981; Wilcott et al., 1976). Sites producing the strongest effects were mainly in the frontal pole and adjacent medial, dorsal, and lateral cortex. The effects of cortical lesions posterior to these electrically mapped regions were also tested.

\section{Method}

Subjects. Thirty-five Holtzman male rats were used. Their weights ranged from 310 to $330 \mathrm{~g}$ at the beginning of the experiment. One rat died immediately after surgery, and two more were dropped because of slow barpressing after surgery.

Surgery. Measurements were made from the bregma and the bone was marked in an attempt to make lesions the same size across all rats. Frontal lesions were intended to begin $4 \mathrm{~mm}$ anterior to the bregma and to extend through the frontal poles. Nonfrontal lesions were intended to cover a rectangular section from $1 \mathrm{~mm}$ anterior to the bregma to $4 \mathrm{~mm}$ posterior, and laterally from $1 \mathrm{~mm}$ to $6 \mathrm{~mm}$ from the bregma. Bone was removed in the marked areas, and the dura was retracted. Cortical tissue was removed by aspiration with a fine pipette. The ventral cortex of frontally lesioned rats was spared because mapping in this area had not been done. Nonfrontal lesions invaded white matter but did not extend below that level. Both operations had been practiced on a number of rats that were immediately sacrificed, their hearts perfused, and their brains removed for examination. The dimensions of cortical lesions of rats used in this experiment were marked on standard charts. Frozen sections $(40 \mu)$ were then prepared and photographic prints made for all frontal rats and about half of the nonfrontal rats.

Sham surgery on control rats was the same as for lesioned rats, except that the dura and cortex were not disturbed.

Rats were maintained on tetracycline in their drinking water for 7 days after surgery.

Barpressing. Three Gerbrands Skinner boxes were used. Each was housed in a standard sound-insulated chamber and controlled by electromechanical programming equipment. Before surgery, the weights of all rats were reduced to $80 \%$ of normal levels, and the rats were shaped to barpress for Noyes food pellets. They were then established on a CRF schedule and given seven daily 15-min sessions. After each session, sufficient food was given in the home cage to maintain weight at the $80 \%$ level. The rats were then separated into three groups that were approximately matched on the basis of barpressing rate during the last three sessions. They were allowed to gain weight with ad-lib feeding for 7 days prior to surgery.

Twenty-one days after surgery, body weights were again reduced to the $80 \%$ level, and barpressing was tested for nine daily 15-min sessions with the CRF schedule. On the 10th day, extinction was begun. Extinction sessions were $20 \mathrm{~min}$ in duration and were given daily for 5 days.

\section{Results}

Lesions. Lesions of the frontal and nonfrontal groups are documented in Figure 1. All of the frontal rats had some damage to the olfactory bulbs, but no damage to the head of the caudate nucleus. Nonfrontal lesions invaded white matter but did not extend below the corpus callosum. Rats were handled daily beginning 7 days after surgery, and they never displayed unusual resistance to being picked up or obvious signs of increased irritability.
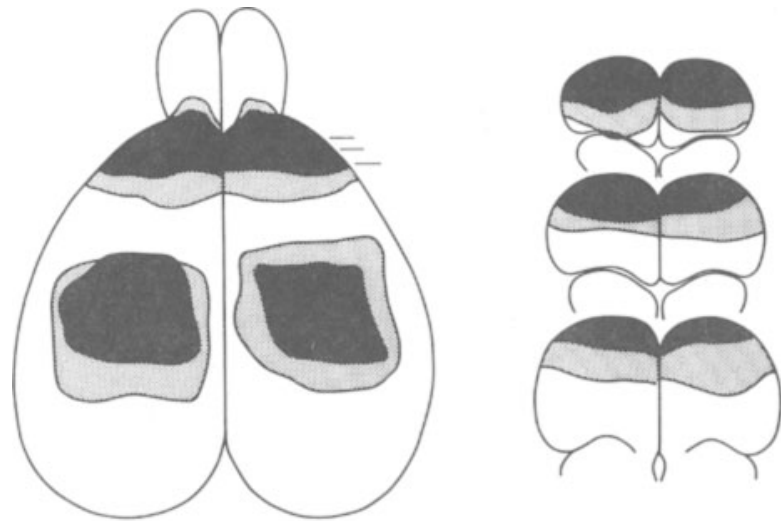

Figure 1. The smallest (black) and largest (stippling) bilateral frontal and nonfrontal cortical lesions. Frontal and nonfrontal lesions of the majority of rats were closer to the smaller size. The approximate locations of the frontal cross sections are marked at the dorsal view.

Barpressing. Mean barpressing rates for the three groups during the last 3 days of CRF presurgical testing and the 9 days of postsurgical testing are given in Figure 2. It is apparent that before surgery the response rates of the three groups were about the same. After surgery there was also little difference between the three groups. An analysis of variance for unequal group size (unweighted means procedure) was performed across Days 1-9 after surgery. Differences in performance between groups were not significant $[\mathrm{F}(2,29)=2.01, \mathrm{p}>.05]$.

Figure 2 also displays the extinction performance of these three groups. Differences between groups across Days 1-5 were not significant $[\mathrm{F}(2,29)=1.79$, $\mathrm{p}>$.05].

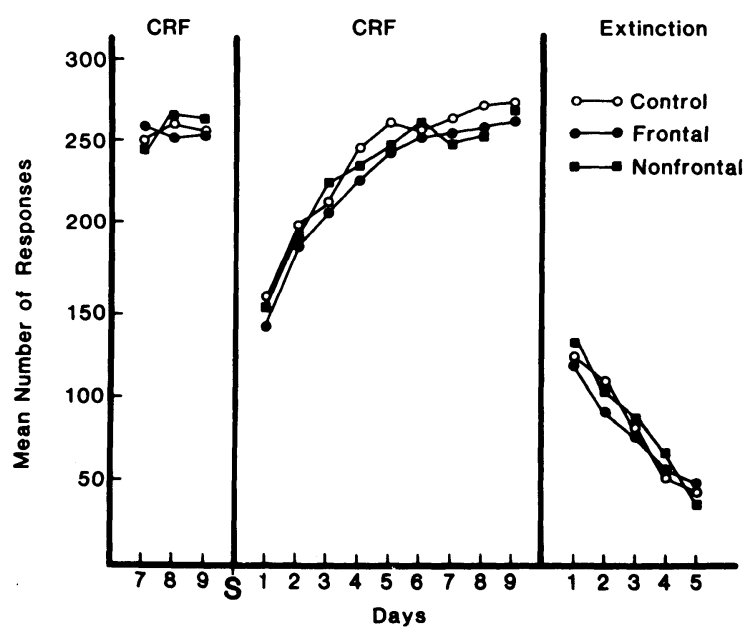

Figure 2. Mean number of barpress responses of the frontal $(N=10)$, nonfrontal $(N=11)$, and control $(N=11)$ groups at the last three CRF trials before surgery (S), and the nine CRF trials and the five extinction trials after surgery. 


\section{EXPERIMENT 2}

The results of Experiment 1 indicate that lesions in parts of the frontal cortex where electrical stimulation will suppress barpressing for food do not have a significant effect on CRF performance or extinction. These findings obviously do not confirm the existence of an inhibitory influence by the frontal cortex on the rate of operant behavior.

A possible confounding factor in Experiment 1, however, could have been the effects of frontal lesions on the activity of the rat. It has been shown that although general activity, as measured in a stabilimeter, is not increased by frontal lesions that include the frontal poles, the general activity of frontal rats is markedly increased by food deprivation (Campbell \& Lynch, 1969). Food deprivation in control rats has little effect on stabilimeter activity. Because the rats in Experiment 1 were hungry at the beginning of daily testing, it appears that response rates of these frontal rats might have been affected by their increased activity.

In Experiment 2, the effects of frontal lesions on the rate of barpressing for food were tested under two conditions, with rats hungry (body weights at the $80 \%$ level) and with ad-lib feeding in the home cage. Preliminary observations demonstrated that trained rats, both frontal and control, will barpress for Noyes pellets even when standard rat food is always available in the home cage, particularly when a continuous reinforcement schedule is used. Extinction was also tested during ad-lib feeding in the home cage.

Stabilimeter activity of frontal and control rats was tested to determine whether the general activity of frontal rats was increased by the magnitude of food deprivation used in these experiments.

\section{Method}

Subjects. Twenty-four Holtzman male rats were used. Their weights ranged from 310 to $325 \mathrm{~g}$ at the beginning of the experiment. One rat died during the course of the experiment. Except as noted, methods were the same as in Experiment 1, including sham operation of controls.

Stabilimeter. Three loudspeaker-type stabilimeters were used. They were the same as those previously described, except that 5-in. speakers were used (Wilcott, 1979). A standard rat cage, fitted with an aluminum cover and resting on metal pegs at each corner, was placed in the center of the stabilimeter platform. The three stabilimeters were placed on a table in a small room, with external noise masked by white noise from a centrally located speaker. Each stabilimeter was coupled to a Beckman dynograph by way of a Beckman EMG integrator. The Beckman was housed in an adjacent room. The magnitude of activity was measured from the integrator recording and specified as the area under the curve (in centimeters squared) as determined by a planimeter (Wilcott, 1979). A predetermined baseline had been marked on the chart at the time of recording.

Procedure. Presurgical testing was not done. Twenty-one days after surgery, the rats were reduced to $80 \%$ of their body weights and were shaped to a CRF schedule. They were then given nine daily 15-min sessions. On each of the last 5 days of testing, and beginning about an hour before the session in the Skinner box, a rat was placed in a stabilimeter for $25 \mathrm{~min}$ of activity recording. Daily recordings were divided into 5 -min intervals, and activity was measured during the last minute of each interval.

Rats were next allowed ad-lib feeding in the home cage for 5 days. Then, with food continuously available in the home cage, they were returned to the Skinner box for nine daily 15-min sessions with a CRF schedule. On the 10th day, extinction trials, $20 \mathrm{~min}$ in duration, were begun; these continued for 6 days.

During the last 5 days of CRF testing with ad-lib feedings, and beginning $1 \mathrm{~h}$ before daily CRF testing, rats were placed in a stabilimeter for $25 \mathrm{~min}$ of activity recording.

\section{Results}

Lesions. The locations and sizes of frontal lesions were approximately the same as in Experiment 1 .

Barpressing. Data for the last 5 days of CRF performance when rats were hungry are given in Figure 3, along with complete data for CRF performance and extinction during ad-lib feeding in the home cage. When rats were hungry, the CRF performance of the frontal groups was not significantly different from that of the control group across Days 5-9 $[F(1,21)=1.99, p>.05]$. Ad-lib home cage feeding also did not produce a greater response rate in the frontal group than in the control group. Differences between groups across Days 1-9 were not significant $[\mathrm{F}(1,21)=1.79, \mathrm{p}>.05]$.

Nevertheless, during extinction with ad-lib home cage feeding, the frontal group responded on Days 16 at a rate significantly higher than that of the control group $[F(1,21)=9.05, p<.01]$. Separate $t$ tests were computed between group means on each day, and differences were significant on Days 1-4 (smallest $\mathrm{t}=2.81, \mathrm{p}<.01$ ) but not on Days 5 and 6 (largest $t=1.13, p>.05)$.

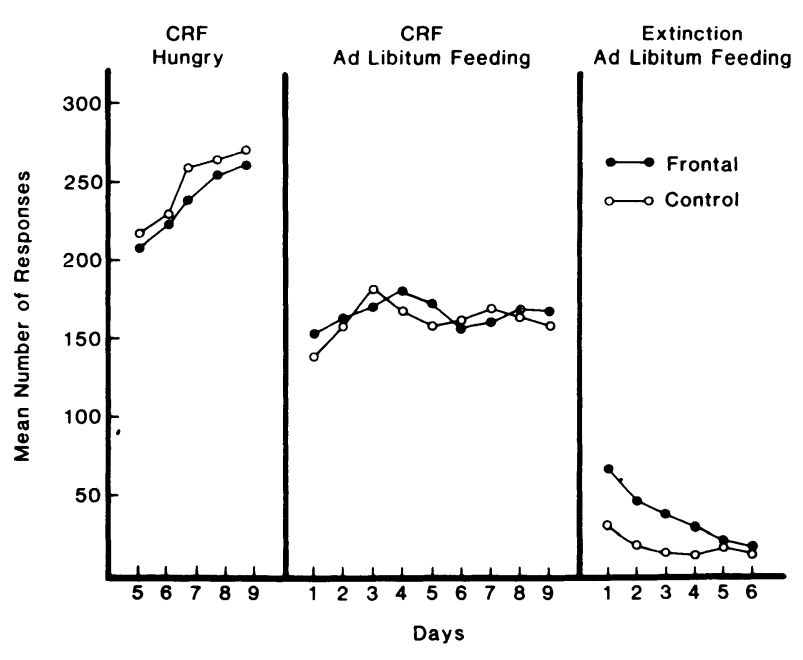

Figure 3. Mean number of barpress responses of frontal $(\mathbf{N}=$ 11) and control $(N=12)$ rats during the last five $C R F$ trials while hungry, the nine CRF trials with ad-lib home cage feeding and the five extinction trials with ad-lib feeding. 


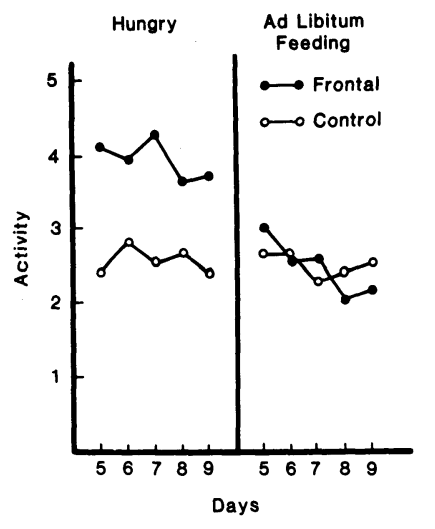

Figure 4. Mean activity score of frontal $(N=11)$ and control $(N=12)$ rats recorded on the last 5 days of CRF testing while hungry, and the last 5 days of CRF testing with ad-lib feeding in the home cage.

The percentage weight gain of the two groups during ad-lib feeding was about the same and did not appear to be a factor in these results. During the first 5 days of ad-lib feeding following CRF training, the mean weight gain was $22 \%$ for the frontal group and $20 \%$ for the control group.

Activity. The relative activity of these two groups during the last 5 days of CRF testing, when the rats were hungry, and the last 5 days of CRF testing, with ad-lib feeding, is given in Figure 4. When hungry, the frontal group was always considerably more active than the control group, and these differences were clearly significant across Days 5-9 $[F(1,21)=12.4$, $\mathrm{p}<.01$ ]. But activity during ad-lib feeding was not significantly different between the two groups across Days 5-9 $[\mathrm{F}(1,21)=1.91, \mathrm{p}>.05]$.

\section{DISCUSSION}

The results of Experiment 1 demonstrated that bilateral lesions in parts of the rat's frontal cortex where electrical stimulation will suppress barpressing for food produced little or no effect on the rate of barpressing with a CRF schedule. The results of Experiment 2 seemed to further demonstrate that this lack of effect on CRF performance was not due to these frontal rats' being hungry. The CRF performance of frontal rats with ad-lib home cage feeding was about the same as that of control rats.

However, the results of Experiments 1 and 2 seem to show that the rate of barpressing during extinction can be influenced by the level of hunger drive. In Experiment 1, hungry frontal rats did not respond more during extinction than did control rats. But in Experiment 2, frontal rats with ad-lib home cage feeding did display greater responding during extinction than control rats. It appears that frontal lesions produced increased response perseveration, or difficulty in terminating an inappropriate response. This effect, however, was not displayed when these rats were hungry. Hunger probably produced greater arousal in frontal rats, which resulted in increased general activity and increased distractibility. The latter may have counteracted or balanced out the tendency for response perseveration.

If hunger in frontal rats produced greater distractibility, the question of why it did not affect the CRF performance of these rats is raised. Increased distractibility should have reduced the rate of responding. But with a CRF schedule, the presence of reinforcement after each response could have served to suppress the distractibility of these rats. This might also explain why hungry frontal rats displayed a reduced rate of responding with a variable-interval schedule (Campbell \& Lynch, 1969). The variability of reinforcement may have actually augmented the distractibility of hungry frontal rats.

The results of this study are consistent with the view that parts of the frontal cortex where stimulation will suppress barpressing for food do have an inhibitory influence on the rate of operant behavior. This influence, however, probably operates only during extinction. But if the inhibitory influence of the frontal cortex is not normally present during reinforced responding, the further question of why stimulation in the frontal cortex suppresses reinforced responding is raised. The answer may be that, while inhibitory cells in the frontal cortex function only under certain conditions, they can be activated by stimulation at any time and forced to display their inhibitory influence on behavior. The stimulation method seems to reveal the existence of these inhibitory cells, but it may not provide an accurate picture of their normal function.

These results seem to disagree with the effects of lesions in the orbital cortex on operant behavior. Hungry orbital rats did respond more during extinction than control rats (Kolb, Nonneman, \& Singh, 1974). But the hunger level of rats in that study may have been less severe than it was in these experiments. Orbital rats were on a 22-h feeding schedule but apparently were given enough food to maintain normal body weights. In this study, hungry rats were allowed only enough food to maintain their body weights at $80 \%$ of previous levels.

\section{REFERENCES}

Campeell, B. A., \& Lynch, G. S. Cortical modulation of spontaneous activity during hunger and thirst. Journal of Comparative and Physiological Psychology, 1969, 67, 15-22.

Glaser, E. M., \& Griffin, J. P. Influence of cerebral cortex on habituation. Journal of Physiology, 1962, 160, 429-445.

Kolb, B., Nonneman, A. J., \& Singh, R. K. Double dissociation of spatial impairments and perseveration following selective prefrontal lesions in rats. Journal of Comparative and Physiological Psychology, 1974, 87, 772-780.

MURRY, M., \& WANG, G. A. Sweating in chronic rostocortical and caudocortical cats. Archives of Italian Biology, 1967, 105, 393-398. 
Schwartz, L. W., \& Isaacson, R. L. Effects of caudate and frontal lesions on acquisition and extinction of an operant response. Physiology \& Behavior, 1972, 9, 155-159.

Siegle, J., \& WANG, R. V. Electroencephalographic, behavioral and single unit effects produced by stimulation of forebrain inhibitory structures in cats. Experimental Neurology, 1974, 42, 28-50.

WANG, G. H., \& Brown, V. W. Suprasegmental inhibition of an autonomic reflex. Journal of Neurophysiology, 1956, 19, 564-572.

Wilcotr, R. C. Cortical control of sweating, skin potential and skin resistance. Psychophysiology, 1967, 4, 500.

Wilcott, R. C. Skeletal and autonomic inhibition from low- frequency electrical stimulation of the cat's brain. Neuropsychologia, 1974, 12, 487-496.

WilcotT, R. C. Cortical stimulation and suppression of activity in the rat. Physiology \& Behavior, 1979, 23, 1041-1048.

WilcotT, R. C. Medial and orbital cortex and the suppression of behavior in the rat. Physiology \& Behavior, 1981, 27, 237-241.

Wilcott, R. C., Sabol, B., \& Yurcheshen, R. P. Frontal cortex and response suppression in the rat. Brain, Behavior and Evolution, 1976, 13, 116-124.

(Manuscript received April 5, 1982;

revision accepted for publication July 2, 1982.) 